\title{
Formations internationales bi diplômantes à l'ENSIAME: Du premier double diplôme européen à la conquête des autres continents
}

\author{
Laurent Cauffriez ${ }^{1}$, Sébastien Grondel ${ }^{2}$, Daniel Coutellier ${ }^{3}$ \\ prénom.nom@univ-valenciennes.fr \\ ${ }^{1}$ Maître de Conférences HDR 61ème, ENSIAME / TEMPO-PSI EA 4542 \\ ${ }^{2}$ Maître de Conférences HDR 63ème, ENSIAME / IEMN-DOAE UMR CNRS 8512 \\ ${ }^{3}$ Professeur, Directeur de l'ENSIAME 60ème / LAMIH FRE CNRS 3304 \\ Ecole Nationale Supérieure d'ingénieurs en Informatique, Automatique, Mécanique, Energétique et Electro- \\ nique \\ Univ. Lille Nord de France - Université de Valenciennes et du Hainaut-Cambrésis \\ Le Mont Houy - 59313 Valenciennes Cedex 9 \\ www.univ-valenciennes.fr/ensiame
}

\begin{abstract}
RESUME : Fortes de l'expérience acquise au cours des 20 dernières années dans le domaine des relations franco-allemandes à travers son réseau d'entreprises, l'ENSIAME, école d'ingénieurs fédérant aujourd'hui l'ensemble des formations d'ingénieurs de l'université de Valenciennes, a ensuite élargi les mobilités académiques dans le cadre de cursus Erasmus et de projets de recherche avec la TU de Dresde et l'université de Sarrebruck. La mobilité en Allemagne a représenté selon les années jusqu'à $70 \%$ de la mobilité internationale de l'école, mobilités académiques et industrielles confondues, ce qui correspond chaque année à un flux d'environ une soixantaine à une centaine d'élèves ingénieurs en cursus académique, en stage de 2ème année, de 3ème année et en Diplôme de Recherche Technologique (DRT). Etre diplômé en France et en Allemagne représente un atout considérable sur le marché du travail et offre une expérience internationale très riche sur les plans scientifiques et humains. Le papier décrit l'organisation du tout premier double diplôme créé à l'ENSIAME avec la TU de Dresde et montrera son adaptation/évolution pour les doubles diplômes montés ensuite avec d'autres universités ou écoles telles que celles de Sarrebruck (Université de la Sarre, Allemagne), de l'école technologique supérieure de Barcelone (ETSEIB, Espagne), de l'université d'Itajuba (Unifei, Brésil), de l'école nationale de Rabat (ENIM, Maroc) et de l'université de Shangaï (Tongji, Chine). Les difficultés rencontrées lors des phases de création mais aussi en régime établi (tels que les flux d'étudiants, la mise à jour des programmes pédagogiques, le passage au LMD, l'évaluation et l'harmonisation des notations) seront soulignées, puis les solutions apportées seront décrites. Enfin, sera présentée l'alternative envisagée par l'ENSIAME avec la création d'un parcours en langue anglaise pour renforcer l'attractivité internationale de l'école et augmenter le flux entrant d'étudiants.
\end{abstract}

Mots clés : Double diplôme, parcours en langue anglaise, transfert de savoir-faire, retour d'expérience.

\section{INTRODUCTION.}

Les relations économiques entre les entreprises françaises et allemandes, qui constituent l'épine dorsale de l'Economie européenne, ne sont plus à prouver. Les deux pays coopèrent de plus en plus étroitement pour pérenniser leurs relations économiques et maintenir leur place dans l'économie mondiale ou la renforcer en commun. Le tout premier double diplôme à l'ENSIAME a été créé, avec comme partenaire, la TU de Dresde.

A l'initiative de l'ENSIAME, les premières réunions de travail relatives à la définition de la convention d'une part et du programme pédagogique d'autre part ont commencé dès février 2000. Cette phase préparatoire a demandé un long travail de négociations pour faire converger les points de vue des deux partenaires (début des négociations en 2000 avec une ouverture effective en 2003), tout en respectant de part et d'autre les contraintes dont celles de la CTI (Commission des Titres d'Ingénieur) du côté français. Ce travail a été initié et développé pour l'ENSIAME par Alain Lecocq, Professeur Certifié en allemand et Laurent Cauffriez, Maître de Conférences HDR en automatique et germanophone de surcroît. Ils ont à eux deux élaboré un modèle de double diplôme européen qui sert aujourd'hui de base à l'école pour la conquête des autres continents. 


\section{CREATION DU PREMIER DOUBLE DIPLOME EUROPEEN AU SEIN DE L'ENSIAME}

\subsection{Organisation du double diplôme et pro- gramme des études}

Ce tout premier double diplôme européen pour l'ENSIAME concernait le domaine du génie mécanique, de la productique et du génie industriel. C'est la filière Informatique et Management des Systèmes (IMS) de l'ENSIAME qui a bénéficié de la primeur avec une ouverture en septembre 2003 d'un double diplôme franco-allemand validé et reconnu par la CTI (Commission des Titres d'Ingénieur). Les élèves ingénieurs de cette filière qui ont opté pour cette formation binationale ont reçu à l'issue de leurs études deux diplômes d'ingénieur: le diplôme d'ingénieur de l'ENSIAME et le diplôme d'ingénieur (TU) de la Technische Universität de Dresde ainsi qu'un certificat de l'université franco-allemande basée à Sarrebruck attestant du caractère binational de ce parcours.

En outre, la volonté commune des deux partenaires était de créer un « programme pédagogique » permettant de conduire à une "promotion binationale » d'élèves français et allemands, afin que les élèves se côtoient, s'entraident et que les enseignements soient identiques en France et en Allemagne.

La durée des études a été prolongée d'un semestre par rapport à la durée normale en école d'ingénieurs (6 semestres) car les contraintes CTI imposent 3 semestres de présence dans l'école pour pouvoir en être diplômé (tout en gardant à l'esprit que les élèves français et allemands doivent faire 2 stages de 6 mois dans l'industrie du pays partenaire durant leurs 7 semestres d'étude). Par ailleurs, il n'apparaissait pas dénué de sens d'étudier un semestre de plus pour obtenir deux diplômes d'ingénieur.

L'organisation pédagogique du double diplôme est donnée figure 1. Le fait que la promotion binationale soit constituée en France, au 5ème semestre, se justifie par le fait que les élèves de l'ENSIAME sont issus du Concours Commun Polytechnique et qu'ils ne pouvaient pas de ce fait partir immédiatement en Allemagne sans que l'école les connaisse, les évalue et les renforce pour une meilleure maîtrise de la langue étrangère. Ceci n'était pas le cas des élèves allemands qui effectuent leur «Vordiplom» (équivalent de nos classes préparatoires) dans les locaux de leur université aux semestres 1 à 4 .

Le programme des études a été défini en langue allemande et française pour chacun des établissements partenaires. Sur le plan scientifique, le double diplôme a pour vocation de donner aux élèves dans les matières mathématiques, scientifiques et des sciences de l'ingénieur, les connaissances, compétences et méthodes nécessaires pour l'exercice de leur activité professionnelle. Par ailleurs, ils doivent être à la hauteur de leurs futures responsabilités dans les domaines économiques, sociaux et écologiques. C'est pourquoi le programme des études comprend en plus des disciplines scientifiques et techniques des matières obligatoires ou optionnelles dans les domaines de la protection de l'environnement, de l'éthique de l'ingénieur, des économies d'énergie, du management et des bases de l'économie d'entreprise. Les étudiants doivent s'ouvrir à la culture socio-économique du pays partenaire et doivent devenir des acteurs majeurs dans leur secteur d'activité et dans le développement des relations francoallemandes. Le double diplôme représente ainsi la base de leur carrière professionnelle comme de leur formation personnelle.

\subsection{Accompagnement et aide aux élèves}

L'accompagnement des élèves (renseignements sur les conditions d'accès à la filière franco-allemande, tâches de conseil sur les inscriptions, choix des matières optionnelles, examens et stages dans l'industrie) est réalisé dans chaque pays partenaire par le responsable en charge du double diplôme et par un tuteur enseignant désigné par l'université d'accueil (dans la pratique, il s'agit souvent de la même personne). Cette mission d'accompagnement des universités partenaires s'élargit à la recherche de stages dans l'industrie (semestres 7 et 11), avec possibilité d'intégrer une formation postingénieur comme le DRT Diplôme de Recherche Technologique [1-2], le sujet du stage étant défini par l'entreprise après concertation de l'université concernée. Ces stages sont sanctionnés par un rapport en langue allemande et française ainsi qu'une soutenance. Il est fréquent qu'à l'issue du stage de fin d'études $\left(11^{\text {ème }}\right.$ semestre) les étudiants soient embauchés dans l'entreprise lorsque celle-ci a été satisfaite du travail réalisé. 


\begin{tabular}{|c|c|c|}
\hline Semestr & $\begin{array}{c}\text { ENSIAME } \\
\text { Valenciennes }\end{array}$ & $\begin{array}{l}\text { TU } \\
\text { Dresde }\end{array}$ \\
\hline 11 & & Stage Industriel* \\
\hline 10 & & Promotion binationale \\
\hline 9 & & Promotion binationale \\
\hline 8 & Promotion binationale & \\
\hline 7 & Stage Industriel* & \\
\hline 6 & Promotion binationale & \\
\hline 5 & Promotion binationale & \\
\hline
\end{tabular}

fig 1 : Organisation pédagogique du tout premier diplôme européen de l'ENSIAME

\subsection{Sélection et évaluation des élèves}

\subsubsection{Sélection des élèves}

La procédure de sélection des candidats comprend les trois phases suivantes :

- Les étudiants doivent justifier de leurs résultats antérieurs, de leurs connaissances linguistiques (niveau $\mathrm{B} 2$ ou $\mathrm{C} 1$ recommandé du Cadre Européen Commun de Référence en langues CECR [3]) et remettre un curriculum vitae en langue allemande et française.

- La sélection est faite par l'université qui accueille (université d'accueil) les élèves de l'université partenaire.

- L'université d'accueil met à la disposition des étudiants sélectionnés les informations et les formulaires nécessaires à leur inscription. Ces formulaires doivent être remplis par les étudiants et renvoyés à la date indiquée par l'université d'accueil avant le début des études.

\subsubsection{Evaluation des élèves}

Les examens ont lieu à la fin de chaque semestre. Ils sont organisés par les universités partenaires qui définissent les règlements des examens, le déroulement des études et la procédure de validation des résultats obtenus. Les examens ont lieu dans le cadre des périodes d'examens et des matières prescrites dans chaque université partenaire. Les étudiants doivent s'inscrire aux examens côté allemand. Les résultats obtenus doivent répondre au règlement des études. De manière générale, les étudiants doivent respecter les règlements académiques définis par l'université d'accueil. Les étudiants ayant échoué lors d'un examen, doivent le repasser dans un délai d'un an.

En ce qui concerne l'évaluation des stages, elle comporte :

- une soutenance à mi-parcours lors de la visite obligatoire en entreprise d'un enseignant tuteur durant la période de stage. Ceci permet à l'enseignant d'avoir un retour de l'industriel et de négocier le placement de stagiaires pour l'année suivante. Lors de cette visite l'étudiant a obligation de présenter le travail réalisé sous la forme d'un exposé dans la langue du pays partenaire. Cette visite représente également l'opportunité pour les universités partenaires de renforcer leurs liens avec les industriels.

- la rédaction d'un mémoire en langue française et allemande,

- la soutenance en fin de stage dans l'université d'accueil devant un jury composé d'enseignants du domaine.

Le jury, qui sanctionne l'ensemble des examens franco-allemands obtenus, est réalisé par une commission académique bilatérale qui a été mise en place, et qui est composée des coordinateurs pédagogiques et institutionnels pour chaque établissement partenaire. Elle a de plus pour mission :

- de vérifier les conditions d'application de la convention du double diplôme,

- de veiller au respect du plan de déroulement des études,

- d'effectuer tous les deux ans un contrôle de l'application de cette convention et de soumettre aux instances compétentes de la faculté (TU Dresde) ou de l'école (ENSIAME) sa prorogation ou sa résiliation.

La commission bilatérale siège en règle générale une fois par an en alternance à Dresde et à Valenciennes.

\subsubsection{Préparation linguistique des élèves}

Trois formes de cours sont proposées pour la préparation linguistique :

- des cours de langue sont offerts à l'université d'origine et sont sanctionnés par un examen. Du côté français, il s'agit d'une formation linguistique intensive en allemand avec trois niveaux de langue (débutant, moyen et confirmé) sanctionné depuis peu par un diplôme de type CLES [4] (certificat de Compétences en Langues de l'Enseignement Supérieur de niveau 1 ou 2),

- dans la mesure du possible, des cours sont offerts par l'université d'accueil, afin que les étudiants de 
l'université partenaire puissent approfondir leurs connaissances linguistiques durant leur séjour.

- les étudiants peuvent également participer à des programmes extérieurs à l'Université : ainsi l'Office Franco-Allemand pour la Jeunesse (OFAJ) [5] accorde, chaque année, des bourses pour cours intensifs d'allemand en Allemagne qui ont pour but la préparation linguistique à un séjour prévu dans l'autre pays. De façon plus globale, les étudiants peuvent également participer au programme CIEL [6] (Cours Intensifs Erasmus de Langues) à condition d'être enregistrés comme étudiants Erasmus [7] (European community for the mobility of university student). Ce programme offre en effet à tout étudiant participant au programme Erasmus la possibilité de suivre entre 3 à 8 semaines de cours intensifs dans le pays d'accueil juste avant leur séjour.

\subsubsection{Aides pratiques et financières}

Les universités soutiennent les étudiants accueillis dans la recherche et l'obtention de bourses. Les aides financières fournies dans le cadre des programmes de l'Union Européenne - en particulier SOCRATES et LEONARDO [7] - et ceux de l'Université FrancoAllemande [8] (UFA-DFHI) sont les plus sollicitées. Les étudiants peuvent aussi bénéficier de bourses nationales et régionales. Aucun frais de scolarité et d'examens n'est perçu par l'université d'accueil, ces frais sont en fait réglés dans l'université d'origine. Les frais correspondant à des cours complémentaires de langue dans le pays d'accueil sont en règle générale à la charge des élèves demandeurs. Des exceptions sont parfois conclues sur la base de la réciprocité. Par ailleurs, l'université d'accueil aide les étudiants inscrits en double diplôme dans leur recherche de logement.

\section{ELARGISSEMENT A LA CREATION D'AUTRES DOUBLE DIPLOMES}

Le schéma de double diplôme franco-allemand avec Dresde présenté dans la première partie a été pris pour modèle et adapté à la création d'un double diplôme avec l'université de Sarrebruck pour la filière mécatronique en 2007 sous la responsabilité de Sébastien Grondel, Maître de conférences HDR en électronique et germanophone. Ce nouveau double diplôme fonctionne suivant le même schéma (voir Figure 1) que le double diplôme de Dresde et favorise ainsi la formation d'une promotion binationale dès le $5^{\text {ème }}$ semestre. Il doit cependant comme nous le verrons au paragraphe 6 évoluer vers une nouvelle organisation en raison du passage au LMD en Allemagne (processus de Bologne).

En revanche, il n'est pas toujours possible selon les parcours des étudiants dans les pays partenaires de favoriser les promotions binationales. En effet, chaque pays a ses modalités spécifiques en ce qui concerne le système d'enseignement supérieur et il est souvent plus facile pour un étudiant étranger d'effectuer son parcours en France en fin de cursus universitaire. Ainsi, ne donnent plus lieu à un cursus organisé sur le principe d'une promotion binationale les doubles diplômes, coordonnés par Daniel Coutellier et Sébastien Grondel, suivants : doubles diplômes avec l'école technologique supérieure de Barcelone (ETSEIB, Espagne, 2004), les universités d'Itajuba (Unifei, Brésil, 2010), de Shangaï (Tongji, Chine, 2010) et l'école nationale de Rabat (ENIM, Maroc, 2010).

\subsection{Du point de vue des élèves étrangers}

L'organisation pédagogique du parcours de double diplôme pour les étudiants étrangers varie quelque peu selon le pays partenaire. Les étudiants de l'ETSEIB réalisent les semestres 5 à 8 comme ceux de la TU de Dresde avant de terminer leur cursus à Barcelone (voir figure 1). Dans le cas du double diplôme avec Unifei (voir figure 2), les étudiants démarrent un semestre plus tôt à l'ENSIAME en raison du décalage des saisons entre l'hémisphère Sud et l'hémisphère Nord. Les étudiants de l'université de Tongji et ceux de l'ENIM doivent d'abord valider leurs huit premiers semestres dans leur université d'origine avant de suivre trois semestres à l'ENSIAME et d'y réaliser ensuite le projet de fin d'études. Il est important de souligner que ce schéma permet à tous les étudiants étrangers de suivre un parcours identique à l'ENSIAME, l'obtention du double diplôme respectant l'ajout d'au moins un semestre supplémentaire par rapport au parcours classique dans le pays partenaire.

\subsection{Du point de vue des élèves français}

L'organisation pédagogique pour les étudiants Français participant à tous les doubles diplômes reste identique à celle indiquée par la Figure 3. Elle implique d'effectuer à l'étranger la troisième année (semestre 9 et 10) ainsi qu'un semestre supplémentaire (semestre 11) condition indispensable à l'obtention du second diplôme. Ce choix découle de la volonté de l'école d'une part, de ne pas multiplier les modèles et d'autre part, de permettre ainsi à tous les étudiants de choisir en $3^{\text {ème }}$ année entre un cursus à l'étranger, un double diplôme, un DRT ou un master.

\section{PRINCIPALES DIFFICULTES A RESOUDRE LORS DE LA MISE EN PLACE DES DOUBLES DIPLOMES}

Les principales difficultés rencontrées lors de mise en place de doubles diplômes sont listées ci-après et ont été classifiées en trois grandes catégories. 


\subsection{Organisation et pédagogie}

Sur les plans organisationnels et pédagogiques, on distingue:

- Le respect des règles de la CTI (Commission des Titres d'Ingénieur) qui demande la présence de 18 mois dans l'école pour en être diplômé.

- La nécessité d'avoir une compatibilité des modules dans chaque pays partenaire tant en volume horaire qu'en contenu.

- Le déroulement du stage imposé dans le pays partenaire.

- La procédure de sélection des élèves candidats: entretiens personnalisés, évaluation du niveau de langue du côté français.

- La notation qui est différente suivant les pays : un module peut être validé à moins de 10 en France alors que des modules peuvent être validés sans note par exemple en Allemagne. En France, l'échelle de notation est de 0 à 20 mais seule la plage 5 à 16 est utilisée alors que du côté allemand, l'échelle de notation est de 1 à 5 et toute l'échelle de notations est utilisée. Le passage à l'ECTS s'est imposé de par ces constats mais aussi par le passage au LMD en Allemagne et l'abandon du titre de Diplom Ing. au profit du diplôme de Master [9].

- La nécessité d'obtenir l'habilitation et l'accréditation des doubles diplômes par les instances des pays partenaires ce qui implique de faire vivre le programme pédagogique du double diplôme suite aux évaluations et recommandations de la CTI du côté français.

\section{Double Diplôme (ENIM)}

\begin{tabular}{|c|c|c|c|c|c|c|}
\hline \multicolumn{2}{|c|}{$3^{\circ \mathrm{I}}$ annuée } & \multicolumn{2}{|c|}{ 4éme arunée } & \multicolumn{2}{|c|}{ Séme aruée } & Semestre \\
\hline $\begin{array}{c}5 \\
\text { Semestre } \\
\text { Pantenaine }\end{array}$ & $\begin{array}{c}6 \\
\text { Semestre } \\
\text { Partenaire }\end{array}$ & $\begin{array}{c}7 \\
\text { Sernestre } \\
\text { Partenaire }\end{array}$ & $\begin{array}{c}8 \\
\text { Semestre } \\
\text { Partenaire }\end{array}$ & $\begin{array}{c}9 \\
\text { Sernestre } \\
\text { ENSIAME } \\
\text { Projet + } \\
\text { cours } \\
\text { électifs }\end{array}$ & $\begin{array}{c}10 \\
\text { Sernestre } \\
\text { ENSIAME } \\
\text { Cours 2 } \\
\text { arruée }\end{array}$ & $\begin{array}{c}11 \\
\text { Sernestre } \\
\text { ENSIAME } \\
\text { Cours } 3^{\text {ime }} \\
\text { arurée }\end{array}$ \\
\hline
\end{tabular}

\section{Double Diplôme (Unifei)}

\begin{tabular}{|c|c|c|c|c|c|c|}
\hline \multicolumn{2}{|c|}{$3^{\circ 1}$ arunée } & \multicolumn{2}{|c|}{ 4éme anuéé } & \multicolumn{2}{|c|}{ Séme aruée } & Semestre \\
\hline $\begin{array}{c}5 \\
\text { Sernestre } \\
\text { Partenaire }\end{array}$ & $\begin{array}{c}6 \\
\text { Semestre } \\
\text { Partenaire }\end{array}$ & $\begin{array}{c}7 \\
\text { Sernestre } \\
\text { Partenaire }\end{array}$ & $\begin{array}{c}8 \\
\text { Sernestre } \\
\text { ENSIAME } \\
\text { Projet + } \\
\text { cours } \\
\text { électifs }\end{array}$ & $\begin{array}{c}9 \\
\text { Semestre } \\
\text { ElNSIAME } \\
\text { Cours 2 } \\
\text { arunée }\end{array}$ & $\begin{array}{c}10 \\
\text { Sernestre } \\
\text { ENSIAME } \\
\text { Cours } 3^{\text {sime }} \\
\text { arriée }\end{array}$ & $\begin{array}{c}11 \\
\text { Sernestre } \\
\text { Stage chez } \\
\text { partenaire ou } \\
\text { en France }\end{array}$ \\
\hline
\end{tabular}

\section{Double Diplôme (Tongji)}

\begin{tabular}{|c|c|c|c|c|c|c|c|c|}
\hline \multicolumn{2}{|c|}{$3^{\circ 1}$ anurée } & \multicolumn{2}{|c|}{ 4éme arunée } & \multicolumn{2}{|c|}{ Séme aruée } & \multicolumn{2}{|c|}{ Géme arunée } & $\begin{array}{c}\text { Semestre } \\
\text { supplémentaire } \\
13 \\
\text { Semestre } \\
\text { Partenaire }\end{array}$ \\
\hline $\begin{array}{c}5 \\
\text { Semestre } \\
\text { Partenaire }\end{array}$ & $\begin{array}{c}6 \\
\text { Sernestre } \\
\text { Partenaire }\end{array}$ & $\begin{array}{c}7 \\
\text { Sernestre } \\
\text { Partenaire }\end{array}$ & $\begin{array}{c}8 \\
\text { Sernestre } \\
\text { Partenaire }\end{array}$ & $\begin{array}{c}9 \\
\text { Sernestre } \\
\text { ENSIAME } \\
\text { Projet + } \\
\text { cours } \\
\text { électifs }\end{array}$ & $\begin{array}{c}10 \\
\text { Sernestre } \\
\text { ENSIAME } \\
\text { Cours 2 } \\
\text { arriée }\end{array}$ & $\begin{array}{c}11 \\
\text { Sernestre } \\
\text { ENSIAME } \\
\text { Cours } 3^{\operatorname{sine}} \\
\text { arnée }\end{array}$ & $\begin{array}{c}12 \\
\text { Sermestre } \\
\text { Partenaire }\end{array}$ & $\begin{array}{c}13 \\
\text { Semestre } \\
\text { Partenaire }\end{array}$ \\
\hline
\end{tabular}

fig 2 : Organisation pédagogique des programmes de double diplôme pour les étudiants étrangers selon le pays 


\section{Cursus classique}

\begin{tabular}{|c|c|c|c|c|c|}
\hline \multicolumn{2}{|c|}{ 1ère année } & \multicolumn{2}{c|}{ 2ème année } & \multicolumn{2}{c|}{ 3ème année } \\
\hline 5 & 6 & 7 & 8 & 9 & 10 \\
Semestre & Semestre & Semestre & Semestre & Semestre & Semestre \\
ENSIAME & ENSIAME & STAGE & ENSIAME & ENSIAME & Stage \\
\hline
\end{tabular}

\section{Double Diplôme

\begin{tabular}{|c|c|c|c|c|c|c|}
\hline \multicolumn{2}{|c|}{ ler année } & \multicolumn{2}{c|}{ 2éme année } & \multicolumn{2}{c|}{ 3éme année } & $\begin{array}{c}\text { Semestre } \\
\text { supplémentaire }\end{array}$ \\
\hline 5 & 6 & 7 & 8 & 9 & 10 & 11 \\
Semestre & Sernestre & Sermestre & Sermestre & Semestre \\
ENSIAME & ENSIAME & STAGE & ENSIAME & $\begin{array}{c}\text { Sermestre } \\
\text { étranger }\end{array}$ & Stage à l'étranger \\
\end{tabular}

fig 3 Organisation pédagogique des programmes classique et de double diplôme pour les étudiants français

- La perception de nos enseignements en école d'ingénieurs qui sont jugés trop lourds et trop scolaires (très nombreux $\mathrm{TD} / \mathrm{TP}$ ) comparativement à certains partenaires.

\subsection{Equilibre entre les flux entrants et sortants}

Le tableau 1 donne les flux entrants et sortants pour les élèves en double diplôme à l'ENSIAME.

\begin{tabular}{|l|c|c|c|c|}
\hline \multicolumn{1}{|c|}{ Pays } & \multicolumn{2}{|c|}{ En cours } & \multicolumn{2}{c|}{ Diplômés } \\
\cline { 2 - 5 } & $\begin{array}{c}\text { Flux } \\
\text { sortant }\end{array}$ & $\begin{array}{c}\text { Flux } \\
\text { entrant }\end{array}$ & $\begin{array}{c}\text { Flux } \\
\text { sortant }\end{array}$ & $\begin{array}{c}\text { Flux } \\
\text { entrant }\end{array}$ \\
\hline Allemagne & 2 & 2 & 5 & 1 \\
\hline Brésil $^{*}$ & 2 & 4 & - & - \\
\hline Chine $^{*}$ & 1 & 1 & - & - \\
\hline Maroc $^{*}$ & 0 & 2 & - & - \\
\hline
\end{tabular}

${ }^{*}$ Pas encore d'élèves diplômés pour ces pays car doubles diplômes récemment créés

Tableau 1: Flux estudiantins en double diplôme à l'ENSIAME

D'une manière générale, au-delà des chiffres, on constate :

- Des contraintes fortes en terme de flux de mobilités estudiantines. Par exemple, flux minimal de 5 élèves français et allemands par année universitaire pour obtenir les bourses et aides au fonctionnement délivrées par l'Université Franco-Allemande (UFADFHI) basée à Sarrebruck.

- Un attrait plus important des élèves issus des pays d'Amérique latine, d'Asie et d'Afrique du Nord que des élèves français pour ces pays (par exemple, au niveau national, on constate pour le Brésil un déséquilibre au niveau du réseau BRAFITEC [10] entre les flux entrants et sortants franco- brésiliens). Néanmoins, le facteur rédhibitoire qui limite la mobilité entrante de ces pays demeure l'obtention de bourses.

- Peu de motivation des élèves français à suivre des études dans des pays étrangers autres qu'anglophones. Pour pallier à cela, l'ENSIAME a décidé d'imposer une seconde langue obligatoire avec nécessité d'obtenir une certification de type C.L.E.S.

\subsection{Langue étrangère pratiquée pour communiquer entre pays partenaires}

Il est impératif que les enseignants impliqués à l'international soient rompus à la pratique des langues étrangères. Sur ce point, parler la langue du pays partenaire est un avantage certain et facilite grandement les choses pour rédiger aisément la convention et le programme pédagogique. Il y a donc nécessité d'avoir de part et d'autre des équipes pédagogiques polyglottes pour faciliter de telles actions à l'international. Il apparaît donc primordial de renforcer le potentiel d'enseignants présentant l'aptitude, du point de vue de la langue mais aussi de la motivation, à être acteur de l'international.

\section{RETOUR D'EXPERIENCE}

Compte tenu de l'aspect novateur, la création du premier double diplôme avec Dresde a été particulièrement difficile et éprouvante pour les acteurs impliqués. En effet, trois années se sont écoulées pour obtenir une version finale de la convention de double diplôme et du programme pédagogique correspondant aux attentes des deux universités partenaires. Ceci a conduit à de nombreux allers-retours des documents et des responsables dans le pays partenaire.

Pour les autres doubles diplômes qui ont suivi, la mise en place a été beaucoup plus rapide. Ceci s'explique notamment par le fait que l'on s'est appuyé sur l'expérience acquise et que le concept initial de formation binationale s'est vu transformé au cas par cas selon les pays partenaires.

\section{PROCESSUS D’EVOLUTION}

Si la création d'un double diplôme fait partie d'un processus long et complexe, son suivi et son évolution ne sont pas pour autant des tâches à négliger. 


\subsection{Passage au LMD}

Le passage au système LMD depuis 2008 pour les universités allemandes en est un exemple concret. Il nécessite de revoir l'organisation pédagogique (voir figure 4) du double diplôme. En effet, les étudiants allemands doivent obtenir à l'issue d'un an d'études en France, un diplôme de licence (Bachelor) à la fin du sixième semestre pour pouvoir continuer leurs études soit en France (double diplôme franco-allemand de Master et d'ingénieur ENSIAME), soit en Allemagne (diplôme de master délivré par l'Allemagne uniquement), ce qui n'était pas le cas auparavant car la réussite aux examens du Vordiplom (correspondant à nos classes préparatoires) suffisait.

\subsection{Suivi et évolution des programmes pédago- giques}

Il convient également de revoir régulièrement les programmes pédagogiques pour que le double diplôme reste attractif. L'évolution des modules doit se faire en relation avec les thèmes porteurs développés dans les instituts de recherche et les universités.
Enfin, la partie communication est un vaste programme. Les outils disponibles pour la communication externe sont nombreux : en premier lieu, la communication dans les universités partenaires (plaquette, poster, présentation, service relations internationales...) est primordiale et nécessite une implication à plein temps des responsables de double diplôme

D'autres voies existent: par exemple, l'Université Franco-Allemande (UFA) joue un rôle essentiel pour les doubles diplômes avec l'Allemagne [8]. Il est également possible pour les écoles d'ingénieurs de s'appuyer sur des organisations comme la CDEFI [10] (Conférence des Directeurs des Ecoles Françaises d'Ingénieurs) et la CGE [11] (Conférence des Grandes Ecoles).

La communication en interne passe quant à elle par l'organisation de journées relations internationales ou de soirées internationales mais surtout par l'intermédiaire des étudiants. Il va sans dire que les étudiants sont les meilleurs ambassadeurs de la promotion d'un double diplôme.

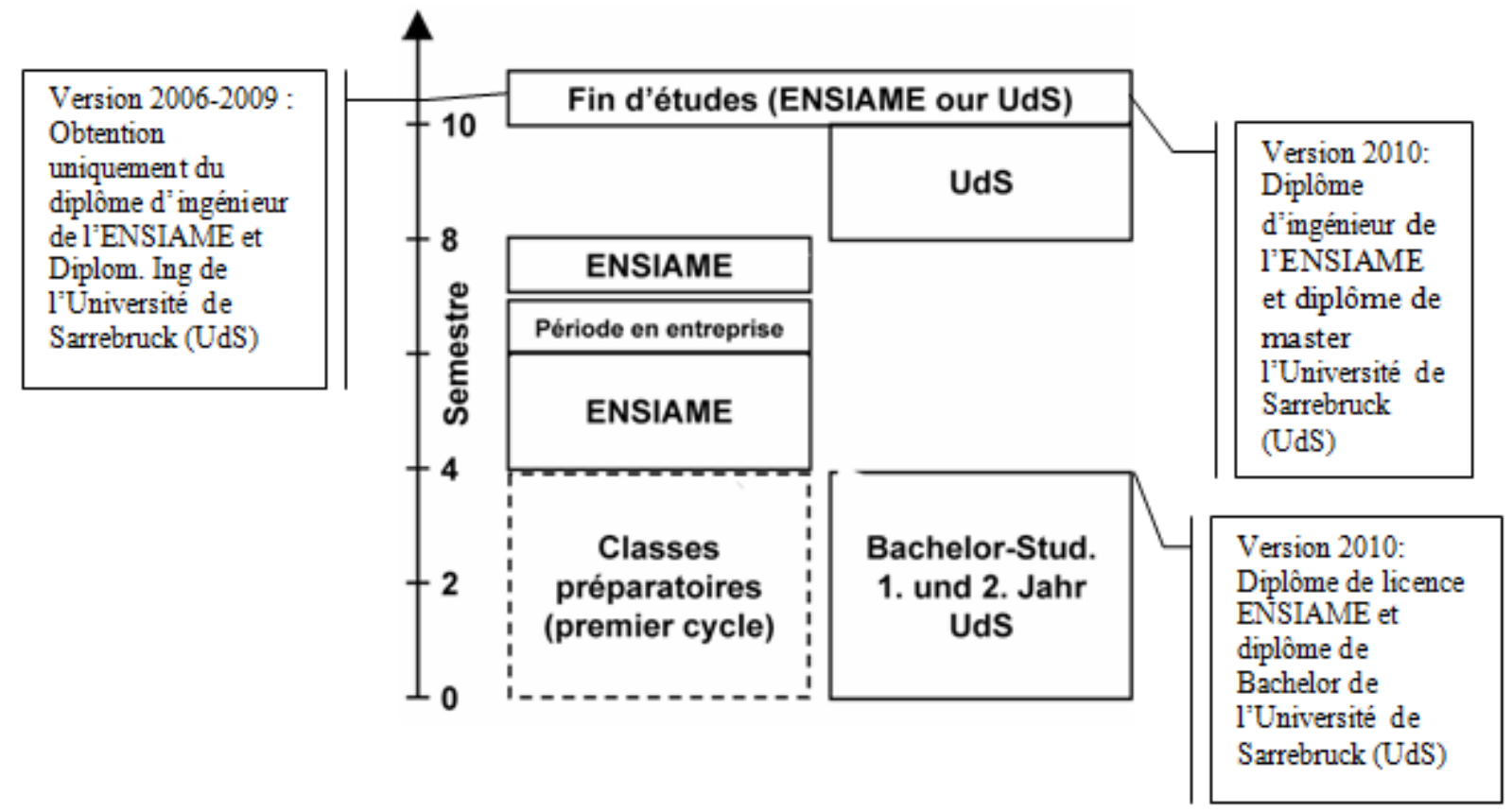

fig 4 Evolution de l'organisation du double diplôme suite au passage du LMD en Allemagne

\section{GENESE DES DOUBLES DIPLOMES}

La genèse d'un double diplôme est un processus qui nécessite au préalable une bonne connaissance des partenaires. Généralement, cela passe par le biais :

- d'accords Erasmus en Europe, de façon à tester et à valider les cursus suivis par les étudiants pour ensuite évoluer vers le double diplôme, c'est le cas du double diplôme avec Dresde et Barcelone,
- d'accords de recherche : le double diplôme de mécatronique avec l'Université de Sarrebruck est lié à l'origine aux accords de recherche entre le DOAE (Département d'opto-acousto-électronique) de l'IEMN (Institut d'Electronique de Microélectronique et Nanotechnologie) [12] et le Fraunhofer de Sarrebruck (IZFP). De même, les accords de double diplôme avec l'ENIM et l'université de Tongji sont apparus grâce à des coopérations de recherches dans les domaines de la production et des transports avec les laboratoires LAMIH et TEMPO [13] [14]. 
- du soutien de programme de coopération et d'échange par les ministères des deux pays partenaires tels que le programme Brafitec (BRAsil FRance Ingénieurs TEChnologie) pour le Brésil.

\section{PERSPECTIVES DE DEVELOPPEMENT}

Pour renforcer l'attractivité internationale et augmenter les flux de mobilité entrants aussi bien anglophones que non anglophones (cf. paragraphe 4.2), l'ENSIAME travaille actuellement à l'ouverture d'un parcours Transports (non diplômant au sens de cursus intégré) dont la particularité est d'avoir des enseignements exclusivement en langue anglaise. Sur ce point, l'école s'appuie sur les compétences et le savoir faire en recherche dans le domaine des transports qui est un point phare de l'université de Valenciennes, renforcé par les acteurs du pôle mondial de compétitivité i-Trans [15] et la création prochaine d'un technopôle sur le campus de Valenciennes reconnu par le Ministère comme campus innovant.

Dans ses perspectives de développement, l'école envisage de transformer ce parcours Transports en diplôme dans le cadre d'un master i (soutenu par le réseau $\mathrm{n}+\mathrm{i}$ qui est un consortium de plus de 70 Grandes Ecoles d'ingénieurs françaises qui ont choisi de mutualiser leurs actions de coopération internationale). Un des avantages du réseau $n+i$ [16] est de pouvoir bénéficier de soutiens financiers (bourses attribuées aux élèves étrangers) et de coopérations industrielles à l'échelle nationale sur la thématique des transports.

\section{CONCLUSION}

Dans cet article, les auteurs ont décrit le processus de conception de double diplôme initialement européen ainsi que son extension à d'autres continents. Les adaptations et évolutions nécessaires selon les pays partenaires ont été ensuite précisées. Les difficultés rencontrées et les solutions adoptées ont été développées.

Les problèmes pédagogiques et organisationnels sont selon nous facilement surmontables avec le bon vouloir des partenaires. A ce jour, les points à conforter demeurent principalement la motivation des élèves et des enseignants à être acteurs de l'international. La nécessité d'inciter les élèves en école d'ingénieurs à obtenir une seconde certification en langue, en plus de l'anglais, reste une question d'actualité. Le recours à une mobilité obligatoire de ces mêmes élèves représente une solution palliative pour ouvrir nos élèves à l'international et les obliger à découvrir d'autres pays et cultures.

L'attractivité de nos formations dispensées sur des thématiques porteuses de manière concomitante en langue anglaise et française (apprentissage de notre langue et de notre culture pour les élèves entrants) représente selon nous une politique d'avenir de développement à l'international.

Sur ce point, on ne peut que souligner l'importance de créer une dynamique autour des contacts recherche et inciter les collègues à une synergie plus forte entre l'enseignement et la recherche au niveau international.

\section{Bibliographie}

[1] "Formation, Grandes écoles", Télérama 2966 du 15 Novembre 2006, p172-173.

[2] "Formation, cours de soutien Séjours linguistiques", Télérama 2967 du 22 Novembre 2006, p174-175.

[3] http://www.coe.int/t/dg4/linguistic/cadre_FR.asp

[4] http://www.certification-cles.fr/

[5] http://www.ofaj.org/

[6] http://www.ef.com.fr/erasmus/

[7] http://www.education.gouv.fr/cid1012/programmeerasmus.html

[8] http://www.dfh-ufa.org/

[9] Durand B., Neubert S., Röseberg D., Viallon V., " Etudier en France et en Allemagne: approche comparée des cultures universitaires ", Presses universitaires Septentrion, ISBN 978-2-75740-021-0

[10] http://www.cdefi.fr/

[11] http://www.cge.asso.fr/

[12] http://www.iemn.univ-lille1.fr/

[13] http://www-univ-valenciennes.fr/LAMIH/

[14] http://www-univ-valenciennes.fr/TEMPO/

[15] http://www.i-trans.org.

[16] http://nplusi.com/.

\section{Curriculum Vitae}

LAURENT CAUFFRIEZ est Maître de Conférences HDR à l'ENSIAME. Il est rattaché à l'équipe Production-ServiceInformation (PSI) du laboratoire TEMPO (Laboratoire Thermique, Ecoulement Mécanique matériaux procédés PrOduction) de l'université de Valenciennes et du HainautCambrésis.

Il s'est vu confier la responsabilité du développement de doubles diplômes avec l'Allemagne de 2000 à 2008 et est à l'origine du premier double diplôme mis en place au sein de l'école. Il a été membre du conseil scientifique de l'Université Franco-Allemande (UFA-DFHI) basée à Sarrebruck de 2001 à 2005.

Il enseigne essentiellement la sûreté de fonctionnement, la sécurité fonctionnelle, le management et la simulation des systèmes de production. Ses activités de recherche ont trait à la sûreté de fonctionnement des systèmes et visent à proposer des méthodes et modèles pour l'évaluation de la sûreté de fonctionnement de systèmes complexes selon une approche systémique. Les champs applicatifs sont les systèmes de production, les systèmes commandés en réseaux et depuis peu, les systèmes de transports guidés dans le cadre de contrats industriels.

SÉBASTIEN GRONDEL est Maître de Conférences HDR à l'ENSIAME. Il est rattaché aux groupes TPIA (Transduction, Propagation et Imagerie Acoustique) et MAMINA (Matériaux et Acoustiques pour MIcro et NAno systèmes intégrés) du département OAE (Optique Acoustique Electronique) de 
l'IEMN (Institut d'Electronique, de microélectronique et de nanotechnologie).

Il s'est vu confier la responsabilité de la création et du suivi du double diplôme avec l'Université de Sarrebruck en 2006 et est responsable depuis 2008 des relations internationales au sein de l'ENSIAME.

Il enseigne essentiellement sur les trois années de la filière mécatronique de l'École d'Ingénieurs ENSIAME. Ses activités de recherche se focalisent plus particulièrement sur la modélisation mécatronique et micro-mécatronique dans le domaine de l'acoustique et du vibratoire. Les champs applicatifs sont le développement de réseaux de transducteurs pour le contrôle non destructif et l'imagerie médicale haute fréquence, la problématique de récupération d'énergie vibratoire dans le domaine des transports et le domaine végétal, ainsi que la réalisation de micro drones à ailes vibrantes.

DANIEL COUTELLIER est Professeur à l'ENSIAME. Il est rattaché à l'équipe Crash Confort et Sécurité (C2S) du LAMIH (Laboratoire d'Automatique, de Mécanique et d'Informatique industrielle et Humaine) de l'Université de Valenciennes et du Hainaut-Cambrésis.

Il a été responsable des relations internationales de l'ENSIAME de 2002 à 2005 et Vice Président de l'Université de Valenciennes et du Hainaut Cambrésis de 2005 à 2010.

Il enseigne essentiellement la méthode des éléments finis pour des applications fortement non-linéaires et en dynamique. Ses activités de recherche ont trait à l'endommagement et la rupture des matériaux principalement composites. Les champs applicatifs sont en majorité les domaines des transports. Parmi les thèses de doctorat encadrées et soutenues, voire en cours, une dizaine est en partenariat avec l'industrie allemande particulièrement avec les grands centres de recherche de Bosch et Daimler. Editeur associé de la revue International Journal for Interactive Design and Manufacturing, il a participé à de nombreux comités scientifiques de congrès internationaux dont certains en tant que président ou vice président. 